\title{
AUTOMATIC PIZZA CUTTING MACHINE
}

\author{
Atif Saeed \\ Lecturer, Department of Mechatronic Engineering, SZABIST, \\ Karachi, (Pakistan).
}

E-mail: m.atif@szabist.edu.pk ORCID: https://orcid.org/0000-0003-1551-4314

Saud Sattar

Postgrad student, MSc Electronics Engineering Management, University of York,

(United Kingdom).

E-mail: saud.sattar@yahoo.com ORCID: https://orcid.org/0000-0003-2477-0838

Andrew Ferguson

Strategic Project Manager/Principal, Careers \& Placement, University of York,

(United Kingdom).

E-mail: andrew.ferguson@york.ac.uk ORCID: https://orcid.org/0000-0003-3146-9330

Recepción: 03/02/2020 Aceptación: 07/04/2020 Publicación: 30/04/2020

Gitación sugerida Suggested citation

Saeed, A., Sattar, S., y Ferguson, A. (2020). Automatic pizza cutting machine. 3C Tecnología. Glosas de innovación aplicadas a la pyme. Edición Especial, Abril2020, 181-193. http://doi.org/10.17993/3ctecno.2020. specialissue5.181-193 


\section{ABSTRACT}

The objective of this research is to determine the need of an automated pizza cutting machine in food industry and to design a machine that would cut a pizza into even slices by a single press. The need of the product has been determined by the surveys that were collected from various pizza vendors. From the survey it was concluded that market will welcome the product as it would overcome several issues like unequal slice cut and lower productivity.

This machine is designed to cut a pizza of any size in less than 30 seconds; increasing the overall productivity. The pneumatic system and round cutting tools are used to create basic structure in this project to get the pizza cuts into even slices with better precision and accurate shape. The stress and motion analysis conducted as part of design procedure and their results are also discussed.

\section{KEYWORDS}

Mechatronics System, Pizza Industry, Productivity, Sustainability, Reliability, CAD Simulations. 


\section{INTRODUCTION}

In today's world as many new technologies have overshadowed mankind's work and as population is increasing day by day. Our needs for products and usage is increasing and everyone wants to get the best of everything as quick as possible. The mission of this project is to design an automatic pizza cutter which cuts pizza into equal number of slices. Optimally each pizza will cut in less than 30 seconds. The aim of this design is to provide an automatic machine in households and in restaurants. As an actuator, pneumatic system is used with a round cutting tool. The cutting tool contains blades, this tool works as a die to cut a pizza.

Knifes and rollerblades often used for cutting purposes in households, industries and meat shops. The process of cutting uses multiple tools, that are operated manually or automatically. The cutting process which uses hands or non-motorized are manually operated tools and the one which are motorized, electrically, hydraulically or pneumatically driven cutting tools are known as automatic or machined tools. There are different types of knives which are available in the market. Daggers of different lengths, width, sizes and weight are available depending on which type of materials or properties we want to cut. Simple single dagger is used in cutting pizza linearly. Multiple dagger can be used to cut pizzas in equal divisions in one single process. This will ensure highest cutting precision. Cutting can be done by round multiple stainless blades (Astakhov \& Davim, 2008).

Metal cutting instruments often have two cutting edges, both oriented to the cutting direction and the tilt continually differs in round-necked instruments. In right-angled cutting, the cutting edge of the work piece is always at the correct angles. When the blade is in the direction of motion it is known as oblique cutting. The slice-push ratio of the blade's velocity or displacement is parallel to the cutting corner displacement and velocity is perpendicular to the cutting corner which makes the cutting easier. The ratio of slice-push is achieved when right-angled blade is driven obliquely as well as inferior (Atkins, 2006; Kurin \& Surdu, 2017). 
Automated cutting devices are commonly used in the processing of various sheets and rolling materials. The materials are cut by a knife, laser, waterjet, plasma or ultrasound controlled by a computer._The most efficient means of cutting textiles are fully automated knife cutting systems._For single-ply and multiply cutting procedures, they guarantee adequately high quality and precision (Deokar et al., 2017; Gracia et al., 2009).

This product aims to increase the speed and efficiency of preparing takeaway and restaurant pizzas globally.

In an article by The Guardian, at the end of 2019 it was noted that Dominos generated a revenue of 339.5 million pounds i.e. 5.5\% more than their sales in 2018. the average market analysis, since 2010 till 2019, shows that this market has an upward trend in turnover and profit (Jolly, 2019). Another article suggested that on average every American spends 23 pounds each year just on Pizza (Links, 2019). Same article on The Guardian quoted that Dominos sold 500,000 pizza solely in UK on Christmas Eve 2019 (Jolly, 2019). In another article it is statistically forecasted that North American pizza market would grow to $10 \%$ in the next 5 years (Littman, 2018). The Economist in an article discussing the pizza industry gave statistics that $83 \%$ of consumers eats pizza at least once a month and as per 2018 industry census, $60.47 \%$ of the respondents reported to increase in sales comparing to their sales of previous year. It also forecasted the growth of $10.7 \%$ in upcoming 5 years (The Economist, 2015). So, any business analyst or entrepreneur can clearly see the potential of this growing market and could readily opt for the machines that could help making things easier for customer facing staff charged with supplying ever increasing numbers of pizzas to customers with increasingly demanding tastes.

The goal of this project is to design and fabricate the automated pizza cutting machine that would function on specific design goals. These design goals were:

- $\quad$ to cut the large pizza up to $12 \mathrm{n}$ into 8 equal slices within 20 seconds,

- to cut the 21-inch pizza into 12 equal slices,

- to implement the safety mechanism on blades so that it shall stop functioning if something is near the blades. 
The project was specifically designed to complete its task in a timely manner and to ensure that slices were of equally distribution so that both the productivity could be increased, and human error of not cutting the slices equally can be overcome. Also, the blades were designed to be covered with butter paper to assure that none of the harmful substances of carbon plated steel could harm food. This coating needs to be replaced after specified cycles in order to assure proper hygiene.

\section{MATERIALS \& METHODS}

\subsection{TURNING IDEAS INTO REALITY}

Firstly the sense, feeling, plans, concept and design have been delivered on the piece of paper. It is the main process in which the whole idea and the theme of the project is based on and hence it takes ample amount of time in getting everything on the right track.

\subsection{RUGGED DRAFT INTO ENGINEERING DESIGN}

Once the rugged drafts and basic designs have been finalized, the whole project goes into the engineering territory and solid modeling have been made. The basic positions, calculation, dimension and placements are considered and get completed in this area of territory.

\subsection{CHOOSING THE RIGHT MATERIAL}

Different shops and markets are there to purchase the specific and appropriate materials for the structure and components of the project. Choosing the right kind of material depends on the application of the material, the environment in which the prototype is tested and the factor of safety of the device.

\subsection{DESIGNING STRUCTURE}

The wooden sheets are carefully dimensioned, cutout, holed and surface is smoothed. The joining of parts has been done by screws

\subsection{MAKING OF BLADE}

The long metals sheets are cut into equal number of sizes shapes and length. All are joined at an angle of 30 degrees between each metal sheets and they are then covered by a thin metal sheet circularly to prevent blades from injuring anyone. 


\subsection{ASSEMBLY OF PARTS}

After designing and production of all parts. The transformation of individual parts into singular device takes place. All the parts are fixed according to their desired geometry and solid model design.

\subsection{PLACING OF PNEUMATIC SYSTEM}

The pneumatic system is placed right into the center of the plate so it will linearly displace along the $y$ axis and cut the pizza evenly from all sides.

\subsection{PLACEMENT OF GAS CYLINDER}

The gas cylinder is placed after all the work is done. It is positioned into the rectangular box. And the pipes are connected to the pneumatic system. The face of the box is closed.

\subsection{CLEANING AND MAINTENANCE OF BLADES}

The system is designed to use a stainless-steel blade which will be nonstick. The device will be easy to handle like any other blender or general use equipment. It is recommended to wash and clean the machine after every 500 cuts. As the blades will be rust free there is no harm in washing them with a standard dishwasher.

The system breakdown is shown in the figure below:

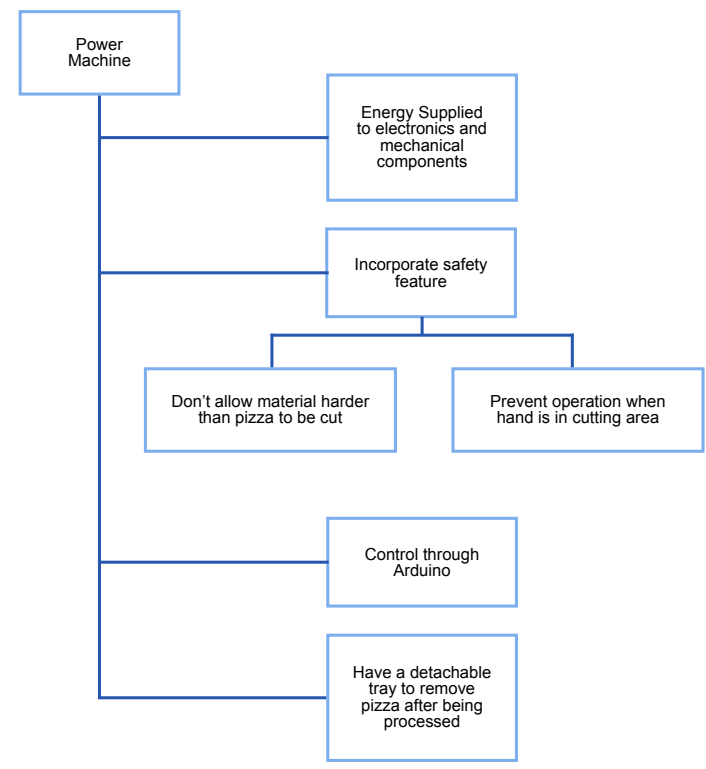

Figure 1. Project Layout. 


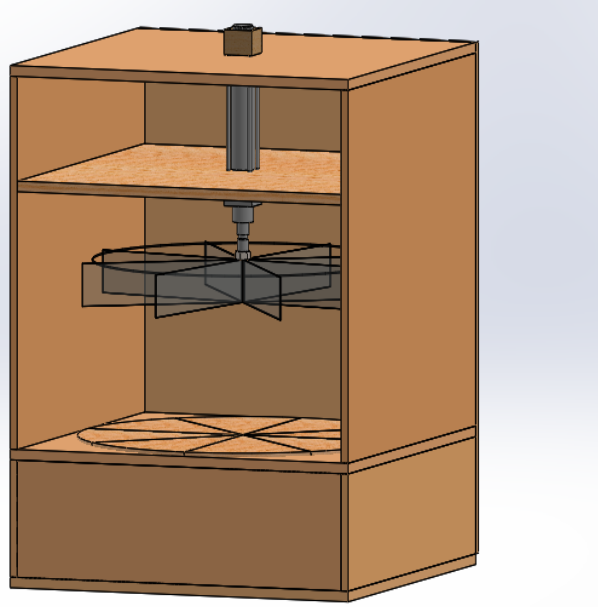

Figure 2. Final Design.

\section{RESULTS}

\subsection{DIMENSION AND MATERIALS}

The dimension of each parts and materials used are mentioned as follows.

Table 1. Dimensions of each material

\begin{tabular}{|c|c|c|c|}
\hline S.no & Name & Material & Dimension \\
\hline 1. & Pneumatic cylinder & Aluminum & $40 \mathrm{~mm}^{*} 150 \mathrm{~mm}$ \\
\hline 2. & Wood & Balsa & $8^{\prime \prime}{ }^{\prime \prime}$ \\
\hline 3. & Screw & Metal & $1 "$ \\
\hline 4. & Blade & Steel & $11 "$ \\
\hline
\end{tabular}

\subsection{STRESS ANALYSIS}

The stress analysis was conducted on medium sized element i.e. not too much fine or coarse. The reason of such mesh selection is the material i.e. wood in this case. The wood which will be used for the prototype is balsa that has high infill density. The load of 5lbf was applied to the pizza dish that pneumatic system will apply to cut the pizza. The yield for the material is $2 \times 10^{7} \mathrm{Kpsi}$ whereas the analysis showed the induced von-mises stress of $7 \times 10^{5}$ Kpsi i.e. below our yielding point. This analysis showed that with given amount of force needed to cut the pizza, the pizza tray wouldn't go for yielding. 


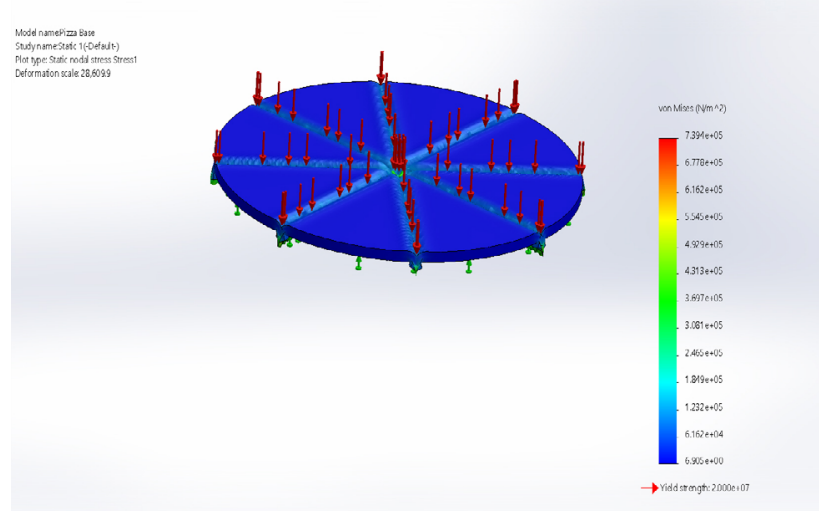

Figure 3. Stress Analysis Results.

\subsection{STRAIN ANALYSIS}
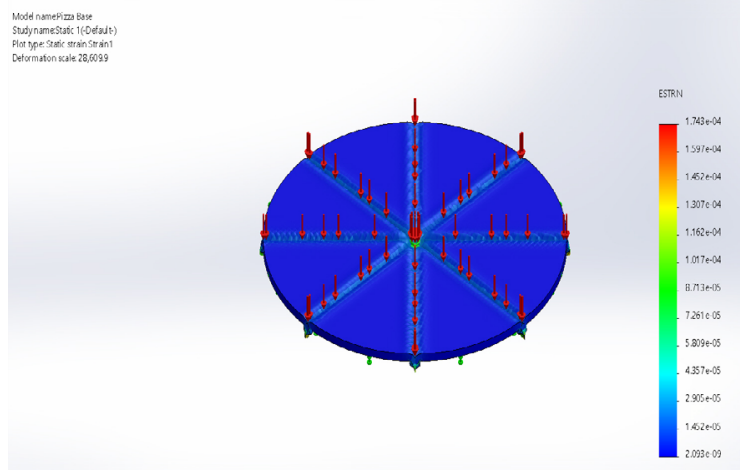

Figure 4. Strain Analysis Results.

The maximum strain of the project is around $1.74 \mathrm{e}^{-4}$.it show that it can bear that amount of force. The factory of safety of that system is 27 .

\subsection{FACTOR OF SAFETY}
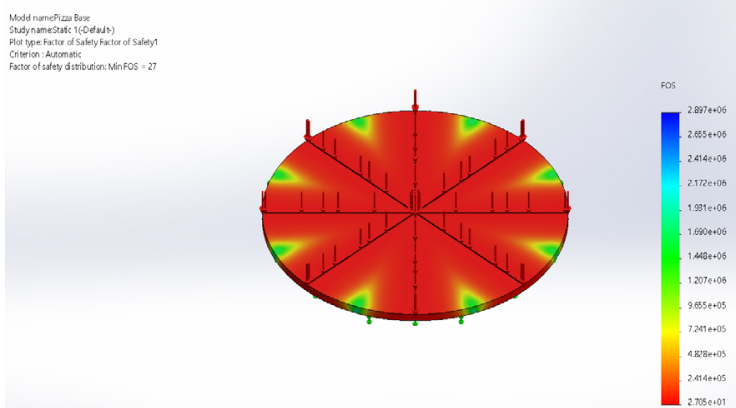

Figure 5. FOS Analysis. 
The factory of safety of this system is 27 . This shows it is safe because the plate will start to break when it reaches to $2.9 * \mathrm{e}^{6}$. It is used as a define factor or as a factor purpose.

\subsection{DISPLACEMENT ANALYSIS}
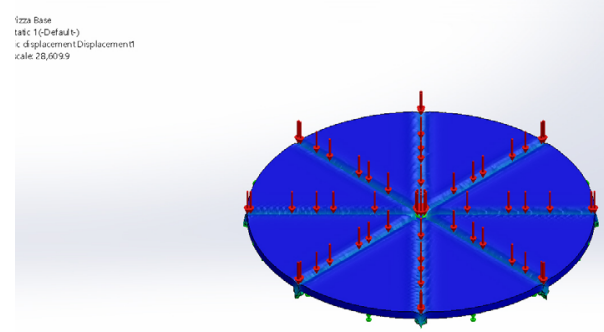

Figure 6. Deflection Analysis.

Designers are often given scenarios where there is a displacement limit in the design, but they either need to find the amount of force needed to reach that displacement or the displacement limit and force is known, but the design needs to be optimized to work within the specific requirements. The maximum displacement of this system is $1.9 * \mathrm{e}^{3}$.

\subsection{MOTION ANALYSIS}

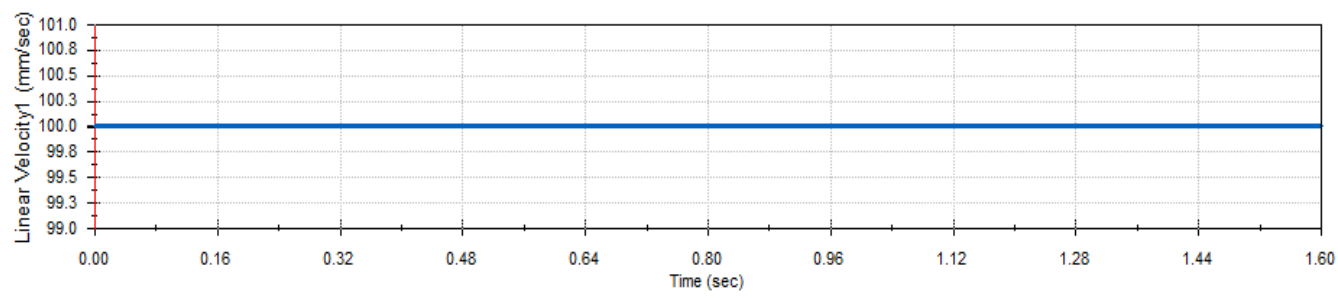

Figure 7. Velocity of cutter prior to contact with pizza.

The cutter puts stress on the plate where the pizza is placed and hence it is the most critical region in our project. The velocity of the system remain contants. 


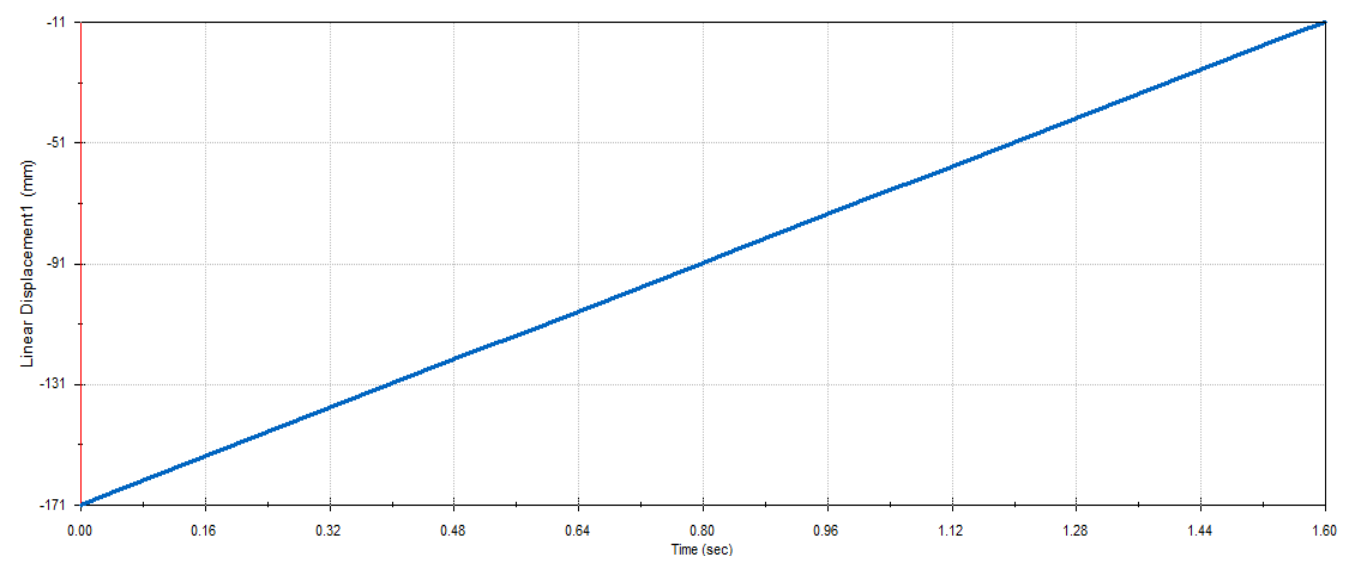

Figure 8. Velocity of cutter after contact with pizza board.

We have seen a linear displacement when we put the motor in the system and study that in motion analysis. The displcaement increases with increase in time. The pneumatic cylinder is linearily diplaced in y direction and cuts the pizza into even slices.

The system can bear more amount of weight if the material is steel or any other type. But the cost factor will also increase with that.

\section{DISCUSSION}

This automatic pizza system can perform specific tasks with better efficiency. The results which we have obtained through our simulation analysis are very close to our expected or practical results. We have put different amounts of pressure and compared the findings with our simulation results. The pneumatic cylinder can provide 150psi pressure. We keep changing the amount of pressure and observe the result obtained. As we can see the stress, strain, displacement and factor of safety in the figures. The plate that we have used for pizza placement contains the most critical region, hence it will not break if we use the pneumatic pressure fully around 150psi.The dimensions and area of the project provide more strength to the system. The system will break if we use a heavier pneumatic system with a higher pressure. Because of this, the system will last longer and will be more efficient as compared to other available systems. 


\subsection{SURVEY ANALYSIS}

As a part of this research, a survey was conducted so that the market need of product could be determined. The survey was forwarded to more than 20 Pizza vendors of different brands. Out of them, 20 pizza vendors responded. The survey consisted of several questions that helped us identify their requirements and expectancy towards this solution. The questions asked are given below.

- Do you face any issues to cut pizza equally?

- Does the unequal pizza slices affects the quality of pizza?

- Do you face any diffculties to serve large number of guests for dine-in and takeway at a time?

- Is their any gap in market to welcome a product that would help the server cutting pizza slices into equal pieces?

- Do the customers complaint regarding the inconsistency of the cut?

Mixed response to this survey were received but most of the responders agreed to the need of such product. Almost, $70 \%$ of the respondents agreed that they normally face issues in cutting the pizza slices equally while $83 \%$ of them agreed that an inconsistent cut affects the overall quality of pizza. Around $78 \%$ of the responders agreed to the fact that during festive seasons they face severe issues in serving the pizza on time that leads to a bad impact on overall companies' image. Also, on an average $73 \%$ of responders agreed that the market will welcome such product that would help the industry in serving their clients better in lesser period of time. This survey clearly yields the need of such product; hence the result of these survey motivates us to move further on to the designing phase.

\section{CONCLUSION}

The automatic pizza cutter can cut the slices into different sizes up to 21 inches. This will help the restaurants to perform tasks quickly and will reduce the cost and time and will add to the precision. Although this project can be considered as a luxury but once used this makes their life easy and be a major factor in reducing time hence turning it into a necessity rather than a luxury. 


\section{ACKNOWLEDGMENTS}

Dr. Tony Ward from the Department of Electronics Engineering. University of York for his constant support.

Dr. Noel Jackson from the Department of Electronics Engineering, University of York for his guidance.

All the pizza companies that volunteered to do the survey.

SZABIST Karachi for providing us labs to do the research.

University of York for financially supporting the research.

\section{REFERENCES}

Astakhov, V., \& Davim,J. (2008). Tools (geometry and material) and tool wear. In Machining: Fundamentals and Recent Advances. Springer, London. https://doi.org/10.1007/978-184800-213-5_2

Atkins, T. (2006). Optimum blade configurations for the cutting of soft solids. Engineering Fracture Mechanics, 73(16), 2523-2531. https://doi.org/10.1016/j. engfracmech.2006.06.006

Deokar, K., Malaviya, K., Mistry, K., Chaudhari, P., \& Dutta, M. (2017). Design and Manufacturing of Coconut De-Husking, Cutting and Grating Machine. International Fournal of Engineering Science and Computing (IFESC), 7(4). http://ijesc.org/upload/ ba58739c0ff3dff6a31 b8ba043180357.Design\%20and\%20Manufacturing\%20 of $\% 20$ Coconut $\% 20$ De-Husking, $\% 20$ Cutting\%20and\%20Grating\%20Machine. pdf

Gracia, L., Perez-Vidal, G., \& Gracia-López, G. (2009). Automated cutting system to obtain the stigmas of the saffron flower. Biosystems Engineering, 104(1), 8-17. https:// doi.org/10.1016/j.biosystemseng:2009.06.003 
Jolly, J. (2019, January 29). Domino's sells more than 500,000 pizzas in record UK trading day. The Guardian. https://www.theguardian.com/business/2019/jan/29/dominos-pizzasrecord-uk-sales-profits

Kurin, M. O., \& Surdu, M. V. (2017). The Concept of the Mechanism and Kinetics of Influence of Mechanochemical Processes on Edge Cutting Machining. Metallofizika i Noveishie Tekhnologii, 39(3). https://doi.org/10.15407/mfint.39.03.0401

Links, Z. (2019, August 01). How Much Pizza Does the Average American Eat in a Year? The Sauce by Slice. https://blog.slicelife.com/how-much-pizza-average-american-eatyear/

Littman, J. (2018, December 17). Report: North American pizza market to grow 10\% in next 5 years. Restaurant Dive. https://www.restaurantdive.com/news/report-northamerican-pizza-market-to-grow-10-in-next-5-years/544523/

The Economist. (2015, February 26). The Economist explains - Rising dough in the pizza industry. https://www.economist.com/the-economist-explains/2015/02/26/rising-dough-in-thepizza-industry 